\title{
The end effector of circadian heart rate variation: the sinoatrial node pacemaker cell
}

\author{
Yael Yaniv ${ }^{1, *} \mathcal{E}$ Edward G. Lakatta ${ }^{2, *}$ \\ ${ }^{1}$ Biomedical Engineering Faculty, Technion-IIT, Haifa, Israel, ${ }^{2}$ Laboratory of Cardiovascular Science, Biomedical Research Center, \\ Intramural Research Program, National Institute on Aging, NIH, Baltimore, Maryland, USA
}

Cardiovascular function is regulated by the rhythmicity of circadian, infradian and ultradian clocks. Specific time scales of different cell types drive their functions: circadian gene regulation at hours scale, activation-inactivation cycles of ion channels at millisecond scales, the heart's beating rate at hundreds of millisecond scales, and low frequency autonomic signaling at cycles of tens of seconds. Heart rate and rhythm are modulated by a hierarchical clock system: autonomic signaling from the brain releases neurotransmitters from the vagus and sympathetic nerves to the heart's pacemaker cells and activate receptors on the cell. These receptors activating ultradian clock functions embedded within pacemaker cells include sarcoplasmic reticulum rhythmic spontaneous $\mathrm{Ca}^{2+}$ cycling, rhythmic ion channel current activation and inactivation, and rhythmic oscillatory mitochondria ATP production. Here we summarize the evidence that intrinsic pacemaker cell mechanisms are the end effector of the hierarchical brain-heart circadian clock system. [BMB Reports 2015; 48(12): 677-684]

\section{INTRODUCTION}

Biological clocks are internal mechanisms that control the periodicity of various biological functions that exist in virtually every life form at every level: single molecules to cells, tissues and organs (for review, cf. (1)). These clock rhythms have time scales that vary between milliseconds to second cycles (ultradian), light and dark cycles (circadian) to longer than

*Corresponding authors. Yael Yaniv, Tel: 972-4-8294124; Fax: 9724-8294599; E-mail: yaely@bm.technion.ac.il, Edward G. Lakatta, Tel: 1-410-558-8202; Fax: 1-410-558-8150; E-mail: lakattae@ grc.nia.nih.gov

http://dx.doi.org/10.5483/BMBRep.2015.48.12.061

Received 1 April 2015, Revised 20 April 2015, Accepted 11 May 2015

Keywords: Cardiac denervation, Coupled-clock pacemaker system, Fractal-like behavior, Heart rate variability, Ultradian rhythm of the heart rate 24-h cycles (infradian) commensurate with their regulated functions. Thus, under normal physiological states, a system of clocks within cells throughout the body creates a highly integrated network of coupled oscillators with a broad range of frequencies to retain sufficient plasticity in response to environmental stimulation. In mammals, the master clocks are located within the brain and broadcast signals to all tissues (2). Specifically, the circadian clock that orchestrates the circadian activity of the entire body is located in the hypothalamic suprachiasmatic nucleus (SCN).

The brain hierarchical clock system modulates heart rate and rhythm via neurotransmitter release from the vagal and sympathetic nerves to the sinoatrial node (SAN), the heart's primary pacemaker, and activates receptors on the pacemaker cell. These receptors activate different internal mechanisms in pacemaker cells. However, the heart has the ability not only to respond to external stimuli, but also possesses internal chemical and electrical clocks within its pacemaker cells (3). Even without external stimuli from the brain to the heart, ultradian rhythms are maintained in the cardiac interval of mammals, including humans (for extensive reviews, see $(4,5)$ ). For example, ultradian rhythms exist in 1) patients following heart transplants (i.e., denervated heart), although there is a shift in their frequency behavior (6); 2) isolated hearts (i.e., when the heart is completely detached from both hormonal and neural input) from mammals, including humans $(7) ; 3)$ monolayers of cultured cells with pacemaker-like activity (8); and 4) isolated rabbit pacemaker cells $(7,9-11)$. It has recently been discovered how internal clock mechanisms control the heart rate and ultradian rhythm (11). The relative role of these mechanisms in the regulation of circadian rhythms is not known. Our review summarizes the evidence that intrinsic pacemaker cell mechanisms are the end effector of the hierarchical circadian clock system.

\section{THE HEART'S PACEMAKER AS PART OF THE CIRCADIAN SYSTEM}

Circadian regulation contributes to normal heart function. For example, certain genes within cardiac cells (e.g., KCNA5, KCND2, KCNK3, KCHIP2) are essential for circadian rhythmic expression in mammals (12, 13), including humans (14). 


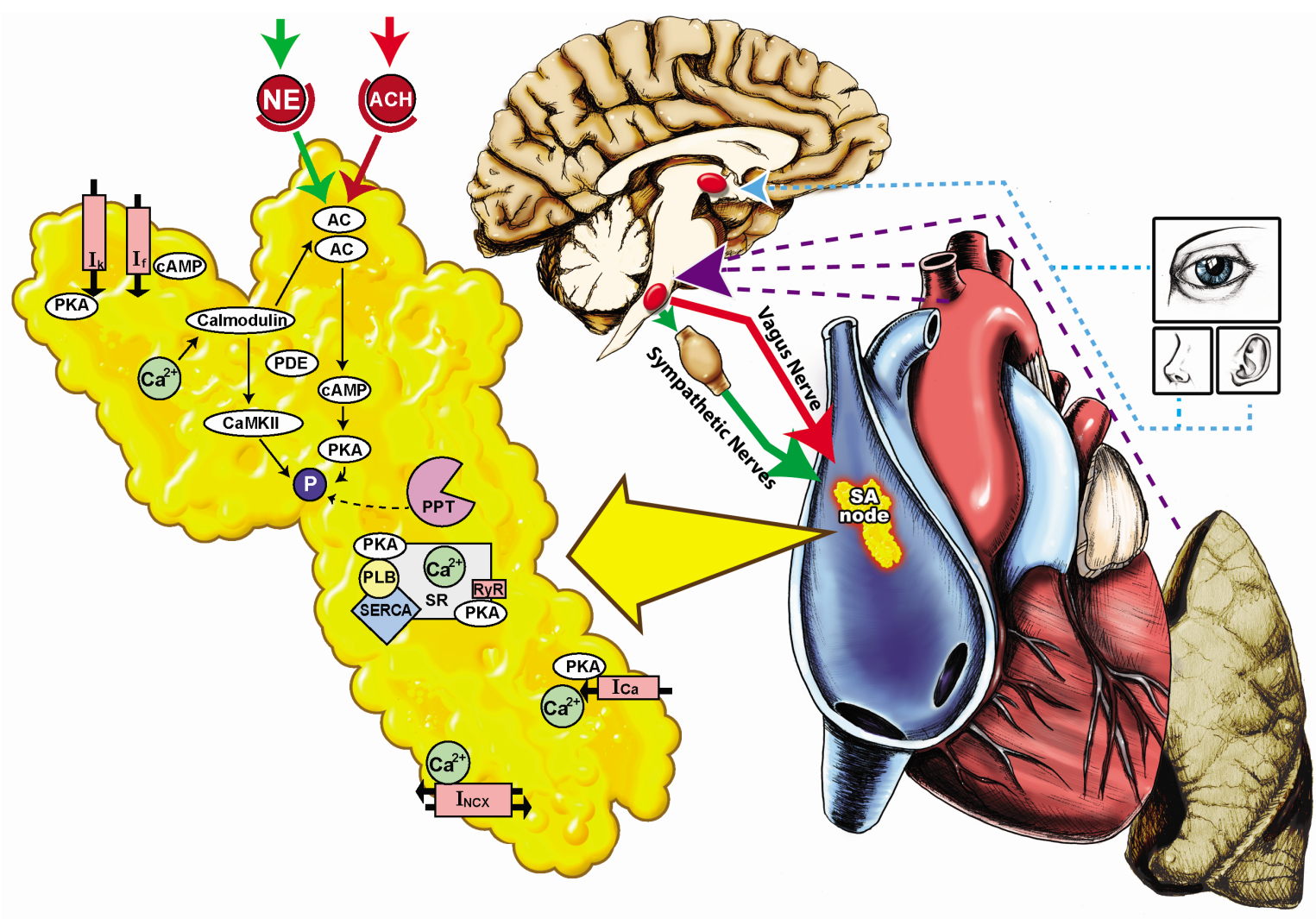

Fig. 1. The heart beat is controlled by a hierarchical clock system (brain-heart) that synchronizes functions of cells within the sinoatrial node (SAN). The circadian system that orchestrates the circadian activity of the entire body is located in the suprachiasmatic nucleus (SCN). The master clock in the $\mathrm{SCN}$, through its anatomical connection with the preautonomic motor neurons in the paraventricular nucleus of the hypothalamus (HyP), transmits signals to the parasympathetic and sympathetic nervous system enforcing its endogenous rhythmicity to the heart and other organs. Different sensory mechanisms (purple line) detect the SCN signaling in the lung and heart: carotid body (CB) chemical receptors, high-pressure baroreceptors (HPBR) and low-pressure baroreceptors (LPBR). Changes in the levels of hormonal influences such as atrial-natriuretic peptides (ANP), and mechanical factors such as atrial pressure are correlated with changes in circadian rhythms. Both the sympathetic and parasympathetic nervous systems innervate the sinoatrial node (red and green lines). The brain hierarchical clock system modulates heart rate and rhythm via neurotransmitter release from the vagal and sympathetic nerves to the SAN. Vagal nerve stimulates cholinergic receptors on the cell membrane (Ch-R) by acetylcholine (ACH), and sympathetic nerve stimulates adrenergic receptor ( $\beta$-AR) also located on the cell membrane by norepinephrine (NE). Even in the absence of neuronal input via receptor stimulation, protein phosphorylation, driven by $\mathrm{Ca}^{2+}$ activation of calmodulin-adenylyl cyclase (AC)-dependent protein kinase $\mathrm{A}$ (PKA) and $\mathrm{Ca}^{2+} /$ calmodulin-dependent protein kinase II (CaMKII) drives cardiac pacemaker sarcoplasmic reticulum (SR) Ca ${ }^{2+}$ cycling proteins (phospholamban (PLB) interacting with sarcoplasmic reticulum $\mathrm{Ca}^{2+}$-ATPase (SERCA), ryanodine receptor (RyR)) and surface membrane ion channels $\left(\mathrm{K}^{+}\right.$current $\left(\mathrm{I}_{\mathrm{k}}\right)$, funny current $\left(\mathrm{I}_{\mathrm{f}}\right), \mathrm{Na}^{+}-\mathrm{Ca}^{2+}$ exchanger $(\mathrm{NCX})$ and L-type current $\left(\mathrm{I}_{\mathrm{Ca}}\right)$ ). Phosphodiesterase (PDE) degrades CAMP and phosphatase (PPT) removes phosphate.

Disruption of such genes in mice leads to cardiac pathology that includes a reduction in average heart rate, increased beating rate variability, and arrhythmias (15). For example, loss of Scn5a circadian gene expression has been documented in mice with cardiomyocyte-specific deletion of Bmal1 (16). Recent evidence suggests that circadian variations within the heart are not only due to autonomic modulation but are also due to intrinsic mechanisms within the heart's pacemaker cells. R-R (the peak of the ventricle activation, QRS complex, of the ECG signal) intervals of patients with transplanted hearts (i.e., the brain-heart signaling via autonomic nerves is not intact) exhibit circadian rhythms (17). However, in these patients the heart rate is higher and the circadian rhythm variation is lower than in hearts with intact autonomic input (18). Similarly, ablation of the sympathetic and parasympathetic inputs in mice disrupts circadian expression of ion channels, but the daily rhythmicity of clock genes within the heart tissue is not completely eliminated (12). Finally, in the isolated working rat heart (i.e., the brain-heart signaling via autonomic nerves is not intact), there are circadian variations in metabolic flux and contractile function (19): "Contractile performance (i.e., cardiac power), carbohydrate oxidation, and oxygen consumption achieve their maxima in the middle of night". 
Mini summary

* Circadian genes are expressed in the heart.

* Disturbances in circadian gene regulation affect heart rate and rhythm.

* Circadian variations exist even without nervous system modulation.

\section{INTERACTIONS BETWEEN CARDIAC AUTONOMIC FUNCTION TO CARDIAC PACEMAKER CELLS AND CIRCADIAN RHYTHM}

The brain hierarchical clock system modulates heart rate and rhythm via neurotransmitter release from the parasympathetic and sympathetic nerves to the SAN (20). Sympathetic or parasympathetic receptor stimulation increases or decreases, respectively, the rate at which nodal cells produce action potentials (APs) $(21,22)$. The level of sympathetic-parasympathetic tone modulates the level of post-translational modification of intrinsic pacemaker cell proteins that drives the coupled-clock mechanism: sympathetic stimulation increases and cholinergic stimulation reduces phosphorylation of proteins that drive coupled-clock molecular functions (i.e., ion channels and $\mathrm{Ca}^{2+}$ pumping to the sarcoplasmic reticulum (SR)) $(22,23)$. The activities of L-type $\mathrm{Ca}^{2+}$ channels, ryanodine channels and the SR-Ca ${ }^{2+}$ pump determine the AP firing rate (for review, cf (3)). Because these mechanism activities are controlled by phosphorylation protein level, the kinetics and stoichiometry of this post-translational protein modification, therefore, can affect the rate and rhythm of spontaneous APs (24). Changes in the sympathetic-parasympathetic tone affect ultradian rhythmic variations: sympathetic stimulation in single pacemaker cell not only markedly decreases the average AP beating interval, but also decreases the ultradian variation indices $(10,25)$. Parasympathetic stimulation, in contrast, not only markedly increases both the average AP beating interval and ultradian AP beating interval variation indices of single isolated pacemaker cells, but also impairs beating interval complexity $(9,10,26)$. Changes in the sympathetic-parasympathetic tone also affect circadian rhythmic variations in mammals including humans (27). Experimental induction of different circadian patterns in humans (shift in the sleep-to-awake transition) demonstrated that "sleep-to-wake transitions occurring in the morning were associated with maximal shifts toward sympathetic autonomic activation compared to those occurring during the rest of the day" (28). Moreover, in both young and aged humans the sympathetic-parasympathetic tone itself is subject to ultradian and circadian rhythmic variations, as evidenced by circadian changes in plasma catecholamine concentrations (29). In a group of healthy young men, both plasma epinephrine and norepinephrine peaked at similar times. Specifically it was demonstrated that "epinephrine levels had no direct relationship to upright posture or rest when awake, whereas norepinephrine levels were significantly higher during upright posture and higher when the subjects were awake than when asleep" (30). If one of the in- puts (i.e., autonomic nervous modulation) to the sinoatrial node system possesses rhythmic behavior, it is not surprising that, by responding to the brain's hierarchical clock signals, or to endogenous molecular clock signals, the sinus node rate in vivo has a circadian rhythm (31) as well as infradian and ultradian rhythms (for review, cf.(4)) (Fig. 1).

\section{Mini summary}

* Both extrinsic and intrinsic mechanisms determine the heart rate.

* Intrinsic and extrinsic mechanisms crosstalk through protein phosphorylation.

* Changes in the sympathetic-parasympathetic tone affect circadian rhythmic variations.

\section{OTHER INPUTS TO PACEMAKER CELLS THAT MEDIATE THE CIRCADIAN RHYTHM}

Even when the brain-heart signaling via autonomic nervous system is not intact, internal signaling expresses circadian variation, for example: 1) Changes in the levels of hormonal influences such atrial-natriuretic and brain-natriuretic peptides (32) are correlated with changes in heart circadian rhythms. Specifically, "correlations between heart circadian rhythm and changes in mRNAs encoding atrial-natriuretic and brain-natriuretic peptides" have been documented in mice (32); 2) circadian variations in mechanical factors may be implicated circadian variation of heart rate. For example, in humans, the circadian pattern of heart rate is correlated with that of atrial pressure (33). Because these hormonal and mechanical mechanisms exist in the denervated or isolated heart, they may also participate in diurnal variations in the heart in vivo (Fig. 1). In parallel to these mechanisms, it is well established that cardiac impulse initiation and conduction involve intracellular $\mathrm{Ca}^{2+}$ and surface membrane electrogenic clocks that are coupled by chemical reactions within cardiac pacemaker cells (34) (for extensive review, see (35)). In the following part of the review, we summarize the evidence that the heart's pacemaker cells are part of the body's circadian system.

\section{Mini summary \\ * Extrinsic mechanisms other than a neural input affect the heart rate and rhythm. \\ * Hormonal and mechanical factors exhibit circadian rhythms. \\ MECHANISMS INTRINSIC TO PACEMAKER CELLS THAT MEDIATE THE CIRCADIAN RHYTHM}

The cardiac pacemaker can be viewed as a series of biological clocks. The interactions among these ultradian clock functions generate spontaneous action potentials (APs). The $\mathrm{Ca}^{2+}$ clock composed of the sarcoplasmic reticulum (SR) that generates local spontaneous ryanodine receptor activation resulting in lo- 
cal diastolic $\mathrm{Ca}^{2+}$ releases that activate an inward $\mathrm{Na}^{+}-\mathrm{Ca}^{2+}$ exchanger current (23). The $\mathrm{Na}^{+}-\mathrm{Ca}^{2+}$ exchanger current, the f-channel current, another member of the ensemble of sarcolemmal electrogenic molecule ensemble ("membrane clock"), and inactivation of $\mathrm{K}^{+}$channels concurrently drive the diastolic membrane depolarization to ignite the next AP. Changes in the membrane potential trigger of activation and inactivation of ionic channels that affect the intracellular $\mathrm{Ca}^{2+}$ balance and consequently the bound $\mathrm{Ca}^{2+}$ to the myofilament and therefore force production. ATP is consumed to maintain these ultradian clock functions. As described above, the ultradian rhythm of heart pacemaker cells is controlled by these coupled-clock mechanisms intrinsic to pacemaker cells and their modulation by autonomic receptor stimulation $(10,11)$. It has recently been shown that the ultradian rhythms of heart rate also exhibit circadian variation. Specifically, in healthy subjects the ratio of low frequency (LF) to high frequency (HF) (36) and HF (37) are greater during the day than at night (in free-running conditions). Moreover, in mammals, including humans, a fractal structure (i.e., self similarity of internal components) of heart rate fluctuations is embedded within the circadian rhythm (38). Therefore, it is tempting to speculate that the same intrinsic coupled-clock mechanisms that control the ultradian rhythms can also regulate circadian rhythms.

Even in the absence of neuronal input via receptor stimulation, protein phosphorylation, driven by $\mathrm{Ca}^{2+}$ activation of calmodulin-adenylyl cyclase (AC)-dependent protein kinase A (PKA) $(39,40)$ and $\mathrm{Ca}^{2+} /$ calmodulin-dependent protein kinase II (CaMKII) drives biophysical mechanisms that couple these clocks to regulate spontaneous AP generation (Fig. 1). In chick retinal cones, CaMKII activity (41), similar to other key cardiac signaling molecules in embryonic chick hearts, including Erk, p38, Akt, and GSK-3 $\beta$ (42), exhibits circadian variation. Because post-translational protein modification kinetics determine the kinetics of the spontaneous AP firing rate, circadian variation of post-translational protein modification kinetics may play a role in the circadian and ultradian rhythms of the cardiac intervals. Future experiments are required to determine whether post-translational protein modification kinetics exhibit circadian rhythms in pacemaker cells similar to those documented in other organs.

Intracellular $\mathrm{Ca}^{2+}$ signaling has been implicated as a key messenger that links the core clock mechanism in SCN neurons to physiological downstream circadian oscillations (43, 44). Specifically, cytosolic $\mathrm{Ca}^{2+}$ and L-type $\mathrm{Ca}^{2+}$ current in mammalian SCN neurons are marked by diurnal and circadian fluctuations (45, 46): "L-type $\mathrm{Ca}^{2+}$ current displays a larger amplitude when the cytosolic $\mathrm{Ca}^{2+}$ increases during the day than the night". Similarly, circadian patterns of regulation of $\mathrm{Ca}^{2+}$ channel subunits, $\mathrm{Ca}^{2+}$ current densities in embryonic chick hearts (42), and $\mathrm{K}^{+}$channel subunit gene expression (47) have been identified in rat hearts. Note that because these experiments were performed in vivo, both intrinsic mechanisms of pacemaker cells and their modulation by the autonomic nerv- ous system can influence the gene circadian patterns. Future research is required to determine whether the beating interval of pacemaker cells exhibits circadian variation linked to circadian variation in intracellular $\mathrm{Ca}^{2+}$, the rate of local $\mathrm{Ca}^{2+}$ release and other coupled-clock functions, and the contribution of these, to circadian variation of heart rate. Note also that circadian patterns in intracellular $\mathrm{Ca}^{2+}$, in turn, in heart pacemaker cells can affect the kinetics of posttranslational protein modification that determine the kinetics of spontaneous AP firing rate (see above).

\section{Mini summary}

* Both $\mathrm{Ca}^{2+}$ and the membrane clock mechanisms crosstalk through phosphorylation.

* Both $\mathrm{Ca}^{2+}$ and the membrane clocks determine the heart rate and ultradian rhythms.

* Future work needs to determine if intrinsic coupled-clock mechanisms that control the ultradian rhythms can also regulate circadian rhythms.

* Future experiments are required to determine whether spontaneous $\mathrm{AP}, \mathrm{Ca}^{2+}$ cycling and post-translational protein modification kinetics exhibit circadian rhythms in pacemaker cells.

\section{CIRCADIAN CLOCKS AND ENERGETIC REGULATION}

There is a circadian pattern of metabolic activity in the rat SCN (48). For example, "glycolysis-related energy pathway activity of SCN is higher during the day than at night" (48). In the heart's pacemaker cells, ATP production by the mitochondria, rather than glycolysis, is largely responsible for maintaining the basal spontaneous AP firing rate (49). Nevertheless, similar to glycolysis, the mitochondrial ATP production exhibits circadian rhythms and the mitochondria can behave as individual pacemakers whose dynamics obey collective network features (50). When the expression of the circadian clock gene CLOCK is altered in the heart, the response of the heart to fatty acid, triglyceride and glycogen metabolism is altered (51). Because ATP supply matches ATP demand on a beat-to-beat basis in the heart (52), generation of ATP with circadian variation may be influenced by and influence the rate and rhythm of ATP consumption. Specifically, future work will have to investigate whether the energy balance of the heart, which is controlled by the key ATP consumer, the sarcomere (53), exhibits circadian rhythms.

In heart pacemaker cells, in addition to regulation of pacemaker AP firing rate, intracellular $\mathrm{Ca}^{2+}$ and $\mathrm{Ca}^{2+}$ activation of CAMP/PKA-CaMKII signaling are the core mechanisms that link utilization of ATP and mitochondrial ATP production (54, 55). SCN cell cultures from rat, in fact, "exhibit robust rhythms in ATP accumulation that are inversely related to the circadian variation in $\mathrm{Ca}^{2+\prime \prime}(56)$. Future experiments in cardiac pacemaker cells are required to verify whether circadian rhythms of the core control mechanisms that link utilization of ATP to the 
mitochondrial ATP production can maintain circadian rhythms of pacemaker cell activity.

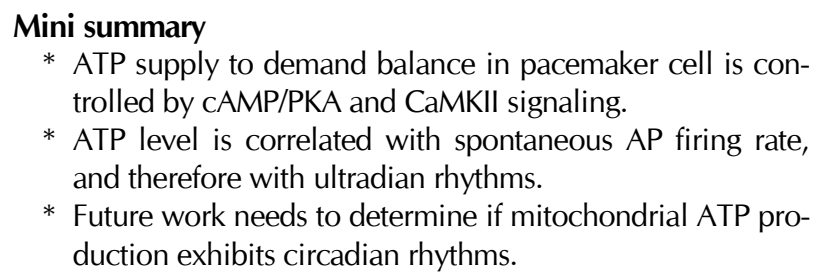

\section{CIRCADIAN CLOCK ALTERATIONS IN HEART DISEASES AND IN AGING}

Elevated heart rate (57) and decreased heart rate variability (i.e. ultradian heart rate rhythm) (58) are both predictors of increased cardiovascular morbidity and mortality. Because of the links between circadian and ultradian rhythms in the heart (see above), it is not surprising that altered circadian rhythms predict increased cardiovascular morbidity and mortality in humans (59). Specifically, increased circadian rhythm variability is associated with carotid stenosis and increases in all measures of coronary atherogenesis (59). Moreover, cardiac disease event occurrence exhibits circadian variation. For example, "different cardiac diseases (coronary ischemic events, acute myocardial infarction and sudden cardiac death) in males appear to peak during transition from sleep, when the cardiac beating interval and the heart rate variability are reduced" (60).

Changes in the properties of coupled-clock mechanisms intrinsic to pacemaker cells that comprise the sinoatrial node and their impaired response to autonomic receptor stimulation have also been implicated in the changes in ultradian rhythms observed in heart diseases $(5,61)$. One can speculate, therefore, that changes in the properties of coupled-clock mechanisms intrinsic to pacemaker cells may also contribute to the changes of circadian rhythm observed in heart diseases.

Patients with chronic heart failure have a higher heart rate and reduced ultradian rhythm compared to healthy subjects (62): Normal circadian rhythms in the ratio of LF to HF do not occur (63). Because of an increase in sympathetic and a decrease in parasympathetic activity in heart failure patients (64), it has been assumed that a modification in the autonomic nervous impulse to pacemaker cells is the major reason for changes in ultradian and circadian rhythms. However, changes in intrinsic mechanisms in pacemaker cells have also been documented in pacemaker cells from chronic heart failure rabbits: "The spontaneous AP firing rate in isolated pacemaker cells of rabbits with heart failure is reduced compared to control (65). Moreover, membrane ionic channel remodeling of pacemaker cells accompanies heart failure (65)". These results indicate that intrinsic clock mechanisms in the pacemaker cells reduce the heart rate and over-compensation by autonomic nervous impulses to reverse this effect leads to an increase in heart rate in vivo.
"In older persons, while circadian variation of heart rate variability is still maintained, the day/night differences are smaller compared to those of younger persons" (66). A parallel reduction in ultradian rhythm has been documented in advanced age (67). Although a reduction in the ratio of parasympathetic to sympathetic tone accompanies advancing age (review in (68)), the age-associated decrease in circadian variation of heart rate variability may, in part, be associated with changes in intrinsic intracellular coupled-clock mechanisms that accompany advanced age. It has been shown recently that advanced age in mice is associated with deficient intrinsic CAMP-PKA-Ca ${ }^{2+}$ signaling of sinoatrial node pacemaker cells (69). Future direct measurements comparing circadian clock rhythms in isolated pacemaker cells from control, heart failure and aged mammals are required to determine whether a reduction in the coupling of intrinsic clock mechanisms is also implicated in disturbances in ultradian and circadian rhythms that have been observed in older persons and in heart failure patients.

\section{Mini summary}

* Deteriorations of intrinsic and extrinsic mechanisms affect the aged-dependent heart rate.

* Altered circadian rhythms predict increased cardiovascular morbidity and mortality in humans.

* Reduction in circadian rhythms accompanies advancing age.

\section{SUMMARY}

In summary, during health and cardiac disease, cardiovascular function is regulated by the rhythmicity of circadian, infradian and ultradian clocks. The ultradian heart rhythm is determined by properties of clocks intrinsic to cardiac pacemaker cells and modulation by the competing influences of the two branches of their autonomic neural input. Virtually no information exists on how core circadian clock gene products interact with the molecular pathways downstream of adrenergic and muscarinic cholinergic receptors in pacemaker cells. The extent to which ultradian clocks regulation of intrinsic SAN cell mechanisms contribute to circadian variations in heart rate awaits further elucidation. This knowledge will contribute greatly to our understanding of cardiac impulse initiation and the specific role of less well understood circadian signaling in pacemaker regulation in health, in aging and in cardiac disease. Because both intrinsic and extrinsic signaling determine circadian and ultradian rhythms, it is impossible to put ones finger on specific mechanism that is involved only in specific rhythm.

\section{ACKNOWLEDGEMENTS}

This research was partially supported by the Intramural Research Program of the $\mathrm{NIH}$, National Institute on Aging and by Technion V.P.R Fund-Krbling Biomedical Engineering 
Research Fund (Y.Y).

\section{REFERENCES}

1. Bell-Pedersen D, Cassone VM, Earnest DJ et al (2005) Circadian rhythms from multiple oscillators: lessons from diverse organisms. Nat Rev Genet 6, 544-556

2. Reppert SM and Weaver DR (2002) Coordination of circadian timing in mammals. Nature 418, 935-941

3. Yaniv Y, Lakatta EG and Maltsev AV (2015) From two competing oscillators to one coupled-clock pacemaker cell system. Front Physiol 6, 47

4. Yaniv Y, Lyashkov AE and Lakatta EG (2013) The fractal-like complexity of heart rate variability beyond neurotransmitters and autonomic receptors: signaling intrinsic to sinoatrial node pacemaker cells. Cardiovasc Pharm Open Access 2, 11-14

5. Yaniv Y, Lyashkov AE and Lakatta EG (2013) Impaired Signaling Intrinsic to Sinoatrial Node Pacemaker Cells Affects Heart Rate Variability during Cardiac Disease. J Clin Trials 4, 2167

6. Bigger JT Jr, Steinman RC, Rolnitzky LM, Fleiss JL, Albrecht P and Cohen RJ (1996) Power law behavior of RR-interval variability in healthy middle-aged persons, patients with recent acute myocardial infarction, and patients with heart transplants. Circulation 93, 2142-2151

7. Monfredi O, Lyashkov AE, Johnsen $A B$ et al (2014) Biophysical Characterization of the Underappreciated and Important Relationship Between Heart Rate Variability and Heart Rate. Hypertension 64, 1334-1343

8. Mandel Y, Weissman A, Schick R et al (2012) Human embryonic and induced pluripotent stem cell-derived cardiomyocytes exhibit beat rate variability and power-law behavior. Circulation 125, 883-893

9. Rocchetti M, Malfatto G, Lombardi F and Zaza A (2000) Role of the input/output relation of sinoatrial myocytes in cholinergic modulation of heart rate variability. J Cardiovasc Electrophysiol 11, 522-530

10. Yaniv Y, Ahmet I, Liu J et al (2014) Synchronization of sinoatrial node pacemaker cell clocks and its autonomic modulation impart complexity to heart beating intervals. Heart Rhythm 11, 1210-1219

11. Yaniv Y, Lyashkov AE, Sirenko S et al (2014) Stochasticity intrinsic to coupled-clock mechanisms underlies beat-tobeat variability of spontaneous action potential firing in sinoatrial node pacemaker cells. I Mol Cell Cardiol 77, 1-10

12. Tong $\mathrm{M}$, Watanabe $\mathrm{E}$, Yamamoto $\mathrm{N}$ et al (2013) Circadian expressions of cardiac ion channel genes in mouse might be associated with the central clock in the SCN but not the peripheral clock in the heart. Biol Rhythm Res 44, 519-530

13. Jeyaraj D, Haldar SM, Wan X et al (2012) Circadian rhythms govern cardiac repolarization and arrhythmogenesis. Nature 483, 96-99

14. Leibetseder V, Humpeler S, Svoboda M et al (2009) Clock genes display rhythmic expression in human hearts. Chronobiol Int 26, 621-636

15. Lakatta EG, Yaniv Y and Maltsev VA (2013) Minding the gaps that link intrinsic circadian clock within the heart to its intrinsic ultradian pacemaker clocks. Focus on "The cardiomyocyte molecular clock, regulation of Scn5a, and arrhythmia susceptibility". Am J Physiol Cell Physiol 304, C941-944

16. Schroder EA, Lefta M, Zhang X et al (2013) The cardiomyocyte molecular clock, regulation of Scn5a, and arrhythmia susceptibility. Am J Physiol Cell Physiol 304, C954-965

17. Kraft IA, Alexander S, Foster D, Leachman RD and Lipscomb HS (1970) Circadian rhythms in human heart homograft. Science 169, 694-696

18. Kotsis VT, Stabouli SV, Pitiriga V et al (2005) Impact of cardiac transplantation in 24 hours circadian blood pressure and heart rate profile. Transplant Proc 37, 2244-2246

19. Young ME, Razeghi P, Cedars AM, Guthrie PH and Taegtmeyer H (2001) Intrinsic diurnal variations in cardiac metabolism and contractile function. Circ Res 89, 1199-1208

20. DiFrancesco D (1993) Pacemaker mechanisms in cardiac tissue. Annu Rev Physiol 55, 455-472

21. DiFrancesco D and Tortora P (1991) Direct activation of cardiac pacemaker channels by intracellular cyclic AMP. Nature 351, 145-147

22. Lyashkov AE, Vinogradova TM, Zahanich I et al (2009) Cholinergic receptor signaling modulates spontaneous firing of sinoatrial nodal cells via integrated effects on PKA-dependent $\mathrm{Ca}(2+)$ cycling and I(KACh). Am J Physiol Heart Circ Physiol 297, H949-959

23. Vinogradova TM, Bogdanov KY and Lakatta EG (2002) beta-Adrenergic stimulation modulates ryanodine receptor $\mathrm{Ca}(2+)$ release during diastolic depolarization to accelerate pacemaker activity in rabbit sinoatrial nodal cells. Circ Res 90, 73-79

24. Yaniv Y, Ganesan A, Yang D, Ziman B, Zhang J and Lakatta EG (2014) Parallel increase in PKA activation kinetics and spontaneous beating rate in sinoatrial node cell in response to chronotropic stimuli. Heart Rhythm 11, S164

25. Zaza A, Robinson RB and DiFrancesco D (1996) Basal responses of the L-type $\mathrm{Ca} 2+$ and hyperpolarization- activated currents to autonomic agonists in the rabbit sino-atrial node. J Physiol 491(Pt 2), 347-355

26. Zaza A and Lombardi F (2001) Autonomic indexes based on the analysis of heart rate variability: a view from the sinus node. Cardiovasc Res 50, 434-442

27. Christ JE (1979) An analysis of circadian rhythmicity of heart rate in tetraplegic human subjects. Paraplegia 17, 251-258

28. Boudreau P, Yeh WH, Dumont GA and Boivin DB (2012) A circadian rhythm in heart rate variability contributes to the increased cardiac sympathovagal response to awakening in the morning. Chronobiol Int 29, 757-768

29. Prinz PN, Halter J, Benedetti C and Raskind M (1979) Circadian variation of plasma catecholamines in young and old men: relation to rapid eye movement and slow wave sleep. J Clin Endocrinol Metab 49, 300-304

30. Linsell CR, Lightman SL, Mullen PE, Brown MJ and Causon RC (1985) Circadian rhythms of epinephrine and norepinephrine in man. J Clin Endocrinol Metab 60, 1210-1215 
31. Cinca J, Moya A, Figueras J, Roma F and Rius J (1986) Circadian variations in the electrical properties of the human heart assessed by sequential bedside electrophysiologic testing. Am Heart J 112, 315-321

32. Goetze JP, Georg B, Jorgensen HL and Fahrenkrug J (2010) Chamber-dependent circadian expression of cardiac natriuretic peptides. Regul Pept 160, 140-145

33. Sato R, Mizuno M, Miura T et al (2013) Angiotensin receptor blockers regulate the synchronization of circadian rhythms in heart rate and blood pressure. J Hypertens 31, 1233-1238

34. Yaniv Y, Sirenko S, Ziman BD, Spurgeon HA, Maltsev VA and Lakatta EG (2013) New evidence for coupled clock regulation of the normal automaticity of sinoatrial nodal pacemaker cells: bradycardic effects of ivabradine are linked to suppression of intracellular $\mathrm{Ca}(2)(+)$ cycling. J Mol Cell Cardiol 62, 80-89

35. Maltsev VA, Yaniv Y, Maltsev AV, Stern MD and Lakatta EG (2014) Modern perspectives on numerical modeling of cardiac pacemaker cell. J Pharmacol Sci 125, 6-38

36. Massin MM, Maeyns K, Withofs N, Ravet F and Gerard P (2000) Circadian rhythm of heart rate and heart rate variability. Arch Dis Child 83, 179-182

37. Bilan A, Witczak A, Palusinski R, Myslinski W and Hanzlik J (2005) Circadian rhythm of spectral indices of heart rate variability in healthy subjects. J Electrocardiol 38, 239-243

38. Hu K, Scheer FA, Buijs RM and Shea SA (2008) The circadian pacemaker generates similar circadian rhythms in the fractal structure of heart rate in humans and rats. Cardiovasc Res 80, 62-68

39. Younes A, Lyashkov AE, Graham D et al (2008) $\mathrm{Ca}(2+)$ -stimulated basal adenylyl cyclase activity localization in membrane lipid microdomains of cardiac sinoatrial nodal pacemaker cells. J Biol Chem 283, 14461-14468

40. Mattick P, Parrington J, Odia E, Simpson A, Collins T and Terrar D (2007) Ca2+-stimulated adenylyl cyclase isoform AC1 is preferentially expressed in guinea-pig sino-atrial node cells and modulates the $\mathrm{I}(\mathrm{f})$ pacemaker current. J Physiol 582, 1195-1203

41. Ko GY, Ko ML and Dryer SE (2001) Circadian regulation of cGMP-gated cationic channels of chick retinal cones. Erk MAP Kinase and Ca2 +/calmodulin-dependent protein kinase II. Neuron 29, 255-266

42. Ko ML, Shi L, Grushin K, Nigussie F and Ko GY (2010) Circadian profiles in the embryonic chick heart: L-type voltage-gated calcium channels and signaling pathways. Chronobiol Int 27, 1673-1696

43. Welsh DK, Takahashi JS and Kay SA (2010) Suprachiasmatic nucleus: cell autonomy and network properties. Annu Rev Physiol 72, 551-577

44. Ikeda M (2004) Calcium dynamics and circadian rhythms in suprachiasmatic nucleus neurons. Neuroscientist 10, 315-324

45. Colwell CS (2000) Circadian modulation of calcium levels in cells in the suprachiasmatic nucleus. Eur J Neurosci 12, 571-576

46. Pennartz CM, de Jeu MT, Bos NP, Schaap J and Geurtsen AM (2002) Diurnal modulation of pacemaker potentials and calcium current in the mammalian circadian clock.
Nature 416, 286-290

47. Yamashita T, Sekiguchi A, Iwasaki YK et al (2003) Circadian variation of cardiac $\mathrm{K}+$ channel gene expression. Circulation 107, 1917-1922

48. Isobe $\mathrm{Y}$, Hida $\mathrm{H}$ and Nishino $\mathrm{H}$ (2011) Circadian rhythm of enolase in suprachiasmatic nucleus depends on mitochondrial function. J Neurosci Res 89, 936-944

49. Yaniv $Y$, Juhaszova $M$, Lyashkov AE, Spurgeon $H$, Sollott SJ and Lakatta EG (2011) Ca2+-regulated-cAMP/PKA signaling in cardiac pacemaker cells links ATP supply to demand. J Mol Cell Cardiol 51, 740-748

50. Aon MA, Cortassa S and O'Rourke B (2006) The fundamental organization of cardiac mitochondria as a network of coupled oscillators. Biophys J 91, 4317-4327

51. Durgan DJ, Trexler NA, Egbejimi O et al (2006) The circadian clock within the cardiomyocyte is essential for responsiveness of the heart to fatty acids. J Biol Chem 281, 24254-24269

52. Yaniv Y, Juhaszova M, Nuss HB et al (2010) Matching ATP supply and demand in mammalian heart In vivo, in vitro, and in silico perspectives. Ann N Y Acad Sci 1188, 133-142

53. Levy C, Ter Keurs HE, Yaniv Y and Landesberg A (2005) The sarcomeric control of energy conversion. Ann N Y Acad Sci 1047, 219-231

54. Yaniv Y, Spurgeon HA, Ziman BD, Lyashkov AE and Lakatta EG (2013) Mechanisms that match ATP supply to demand in cardiac pacemaker cells during high ATP demand. Am J Physiol Heart Circ Physiol 304, H1428-1438

55. Yaniv Y, Spurgeon HA, Ziman BD and Lakatta EG (2013) $\mathrm{Ca}(2)+/$ calmodulin-dependent protein kinase II (CaMKII) activity and sinoatrial nodal pacemaker cell energetics. PLoS One 8, e57079

56. Burkeen JF, Womac AD, Earnest DJ and Zoran MJ (2011) Mitochondrial calcium signaling mediates rhythmic extracellular ATP accumulation in suprachiasmatic nucleus astrocytes. J Neurosci 31, 8432-8440

57. Custodis F, Reil JC, Laufs U and Bohm M (2013) Heart rate: a global target for cardiovascular disease and therapy along the cardiovascular disease continuum. J Cardiol 62, 183-187

58. Hillebrand S, Gast KB, de Mutsert R et al (2013) Heart rate variability and first cardiovascular event in populations without known cardiovascular disease: meta-analysis and dose-response meta-regression. Europace 15, 742-749

59. Bassiouny HS, Zarins CK, Lee DC, Skelly CL, Fortunato JE and Glagov S (2002) Diurnal heart rate reactivity: a predictor of severity of experimental coronary and carotid atherosclerosis. J Cardiovasc Risk 9, 331-338

60. Furlan R, Barbic F, Piazza S, Tinelli $M$, Seghizzi $P$ and Malliani A (2000) Modifications of cardiac autonomic profile associated with a shift schedule of work. Circulation 102, 1912-1916

61. Yaniv Y, Tsutsui K and Lakatta EG (2015) Potential effects of intrinsic heart pacemaker cell mechanisms on dysrhythmic cardiac action potential firing. Front Physiol 6, 1-7

62. Rydlewska A, Jankowska EA, Ponikowska B, Borodulin-Nadzieja L, Banasiak W and Ponikowski P (2011) Changes in autonomic balance in patients with de- 
compensated chronic heart failure. Clin Auton Res 21, 47-54

63. Wu GQ, Shen LL, Tang DK, Zheng DA and Poon CS (2006) Circadian rhythms of spectral components of heart rate variability. Conf Proc IEEE Eng Med Biol Soc 1, 3557-3560

64. Peng CK, Havlin S, Hausdorff JM, Mietus JE, Stanley HE and Goldberger AL (1995) Fractal mechanisms and heart rate dynamics. Long-range correlations and their breakdown with disease. J Electrocardiol 28 Suppl, 59-65

65. Verkerk AO, Wilders R, Coronel R, Ravesloot JH and Verheijck EE (2003) lonic remodeling of sinoatrial node cells by heart failure. Circulation 108, 760-766

66. Burger AJ, Charlamb M and Sherman HB (1999) Circadian patterns of heart rate variability in normals, chronic stable angina and diabetes mellitus. Int J Cardiol 71, 41-48

67. Pikkujamsa SM, Makikallio TH, Sourander LB et al (1999) Cardiac interbeat interval dynamics from childhood to senescence : comparison of conventional and new measures based on fractals and chaos theory. Circulation 100, 393-399

68. Santulli G and laccarino G (2013) Pinpointing beta adrenergic receptor in ageing pathophysiology: victim or executioner? Evidence from crime scenes. Immun Ageing 10,10

69. Liu J, Sirenko S, Juhaszova M et al (2014) Age-associated abnormalities of intrinsic automaticity of sinoatrial nodal cells are linked to deficient CAMP-PKA-Ca(2+) signaling. Am J Physiol Heart Circ Physiol 306, H1385-1397 